\title{
A New Particle Swarm Optimization Algorithm Incorporating Reproduction Operator for Solving Global Optimization Problems
}

\author{
Millie Pant ${ }^{1}$, Radha Thangaraj ${ }^{1}$ and Ajith Abraham ${ }^{2}$ \\ ${ }^{1}$ Department. of Paper Techology, IIT Roorkee, India \\ ${ }^{2}$ Center of Excellence for Quantifiable Quality of Service, \\ Norwegian University of Science and Technology, Norway \\ millifpt@iitr.ernet.in,t.radha@ieee.org,ajith.abraham@ieee.org
}

\begin{abstract}
This paper presents a new variant of Basic Particle Swarm Optimization (BPSO) algorithm named QI-PSO for solving global optimization problems. The QI-PSO algorithm makes use of a multiparent, quadratic crossover/reproduction operator defined by us in the BPSO algorithm. The proposed algorithm is compared it with BPSO and the numerical results show that QIPSO outperforms the BPSO algorithm in all the sixteen cases taken in this study.
\end{abstract}

\section{Introduction}

Evolutionary Algorithms (EA) and Particle Swarm Optimization (PSO) are perhaps the two most common population based, stochastic search techniques for solving continuous global optimization problems. Both the techniques have been quite efficient in solving complex optimization problems (test cases as well as real life problems). On one hand, EA are inspired from the metaphor of natural biological evolution using the concepts of selection, mutation and reproduction. On the other hand, PSO uses the complex social cooperative and competitive behavior exhibited by different species like birds, bees humans etc. some of the similarities between the EA and PSO as pointed out by Angeline [1] may be given as:

$>$ Both are population based search techniques.

$>$ Neither requires the auxiliary knowledge of the problem.

> In both the algorithms, solutions belonging to the same population interact with each other during the search process to move towards the better optima.

$>$ The quality of the solutions are improved using techniques inspired from real world phenomenon like human genetics in case of EA and cooperative behavior in case of PSO.

Both the algorithms have their share of weaknesses and strengths and One of the simplest methods to enhance the performance of both the algorithms is to exploit the strengths of both the algorithms together in a single algorithm. A number of techniques suggesting a combo of these two methods are available in literature. Out of the EA operators' viz. selection, crossover and mutation, the one that has been used most frequently is the mutation operator. Some of the commonly used mutation operators used in PSO algorithms are Gaussian, Cauchy, linear, nonlinear operators etc. For a detailed description, the reader is suggested [2] - [7]. However, for selection and reproduction operator only a few examples are available (see for instance Angeline [8], Clerc [9]).

In this paper we have suggested the use of a new crossover operator named quadratic crossover operator in a BPSO algorithm. The most potent particle (one having the lowest objective function value) is selected as the leader of the swarm (tribe). The leader is then allowed to mate with two other randomly chosen particles of the swarm (tribe). The child (or new particle) lies at the point of minima of the quadratic curve passing through the three (different) chosen particles (leader and two mates).

The remaining paper is structured as follows: in Section 2, we have briefly explained the Basic Particle Swarm Optimization, in Section 3; we have defined the proposed QI-PSO algorithm. Section 4 deals with experimental settings. Section 5 gives numerical results and finally the paper concludes with Section 6 . 


\section{Basic Particle Swarm Optimization}

PSO is a relatively newer addition to a class of population based search technique for solving numerical optimization problems. The particles or members of the swarm fly through a multidimensional search space looking for a potential solution. Each particle adjusts its position in the search space from time to time according to the flying experience of its own and of its neighbors (or colleagues).

For a D-dimensional search space the position of the ith particle is represented as $X_{i}=\left(x_{i 1}, x_{i 2}, \ldots, x_{i D}\right)$. Each particle maintains a memory of its previous best position $\mathrm{P}_{\text {besti }}=\left(\mathrm{p}_{\mathrm{i} 1}, \mathrm{p}_{\mathrm{i} 2} \ldots \mathrm{p}_{\mathrm{iD}}\right)$. The best one among all the particles in the population is represented as $P_{\text {gbest }}=$ $\left(\mathrm{p}_{\mathrm{g} 1}, \mathrm{p}_{\mathrm{g} 2} \ldots \mathrm{p}_{\mathrm{gD}}\right)$. The velocity of each particle is represented as $V_{i}=\left(v_{i 1}, v_{i 2}, \ldots v_{i D}\right)$. In each iteration, the $\mathrm{P}$ vector of the particle with best fitness in the local neighborhood, designated $\mathrm{g}$, and the $\mathrm{P}$ vector of the current particle are combined to adjust the velocity along each dimension and a new position of the particle is determined using that velocity. The two basic equations which govern the working of PSO are that of velocity vector and position vector given by:

$$
\begin{aligned}
& v_{i d}=w v_{i d}+c_{1} r_{1}\left(p_{i d}-x_{i d}\right)+c_{2} r_{2}\left(p_{g d}-x_{i d}\right) \\
& x_{i d}=x_{i d}+v_{i d}
\end{aligned}
$$

The first part of (1) represents the inertia of the previous velocity, the second part is the cognition part and it tells us about the personal experience of the particle, the third part represents the cooperation among particles and is therefore named as the social component [10]. Acceleration constants $c_{1}, c_{2}$ [11] and inertia weight $w[12]$ are the predefined by the user and $r_{1}, r_{2}$ are the uniformly generated random numbers in the range of $[0,1]$. The computational steps of BPSO algorithm are illustrated below.

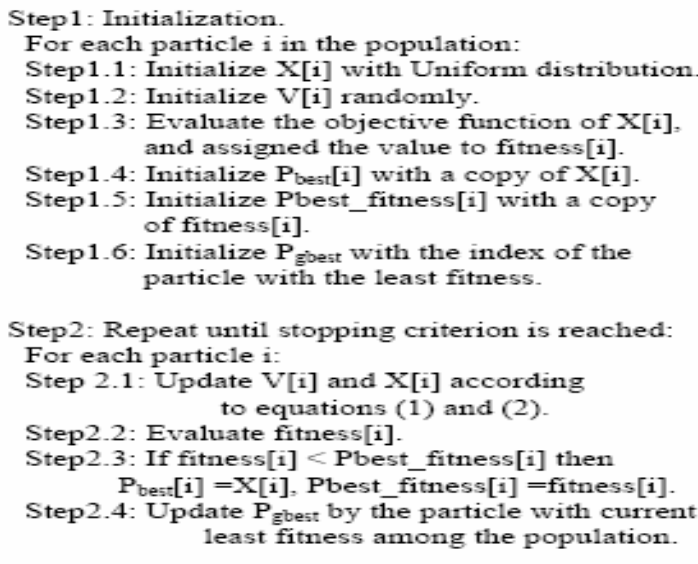

\section{Proposed QI-PSO Algorithm}

The proposed QI-PSO algorithm is a simple and modified version of BPSO in which we have induced the concept of reproduction (borrowed from EA).

The basic idea of this paper is loosely based on the concept of polygamy (still prevalent in some of the tribal regions of India), where the leader of the tribe is allowed to have more than one partner. The QI-PSO algorithm works in the following manner. In every iteration, a leader of the swarm (tribe) is selected according to the performance and the one giving the least function value is taken to be the leader of the tribe. Once the leader is elected, then its partners (or mates) are chosen randomly from the remaining members of the tribe. For producing a new offspring from the union of three parents, we have defined a new crossover operator.

The quadratic crossover operator, based on quadratic interpolation [13], suggested in this paper is a nonlinear crossover operator which produces an offspring lying at the minimum of the quadratic curve passing through the three selected parents. The QI-PSO algorithm starts like the usual BPSO using equations (1) and (2). After that the crossover operator is invoked and the new particle is accepted in the swarm only if it is better than the worst particle present in the swarm. The process is repeated iteratively until a better solution is obtained. It uses $\mathrm{a}=\mathrm{X}_{\min }$, (the leader having minimum function value) and two other randomly selected particles $\{b, c\}$ $(\mathrm{a}, \mathrm{b}$ and $\mathrm{c}$ are different particles) from the swarm (tribe) to determine the coordinates of the new particle $\tilde{x}^{i}=\left(\tilde{x}^{1}, \tilde{x}^{2}, \ldots \ldots . \tilde{x}^{n}\right)$, where

$$
\tilde{x}^{i}=\frac{1}{2} \frac{\left(b^{i^{2}}-c^{i^{2}}\right) * f(a)+\left(c^{i^{2}}-a^{i^{2}}\right) * f(b)+\left(a^{i^{2}}-b^{i^{2}}\right) * f(c)}{\left(b^{i}-c^{i}\right) * f(a)+\left(c^{i}-a^{i}\right) * f(b)+\left(a^{i}-b^{i}\right) * f(c)}
$$

The nonlinear nature of the quadratic crossover operator used in this work helps in finding a better solution in the search space. The computational steps of the algorithm are illustrated below.

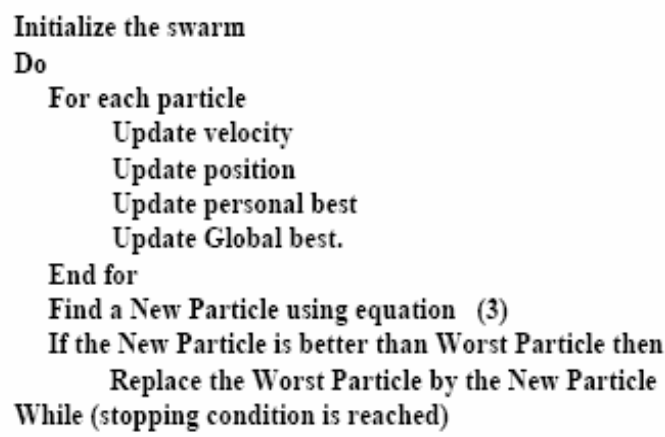


Table 1. Numerical benchmark problems

\begin{tabular}{|c|c|c|c|}
\hline Function & Dim & Range & $\begin{array}{l}\text { Min. } \\
\text { value }\end{array}$ \\
\hline$f_{1}(x)=\sum_{i=1}^{n}\left(x_{i}^{2}-10 \cos \left(2 \pi x_{i}\right)+10\right)$ & $30 / 50$ & {$[-5.12,5.12]$} & 0 \\
\hline$f_{2}(x)=\sum_{i=1}^{n} x_{i}^{2}$ & $30 / 50$ & {$[-5.12,5.12]$} & 0 \\
\hline$f_{3}(x)=\frac{1}{4000} \sum_{i=0}^{n-1} x_{i}^{2}-\sum_{i=0}^{n-1} \cos \left(\frac{x_{i}}{\sqrt{i+1}}\right)+1$ & $30 / 50$ & {$[-600,600]$} & 0 \\
\hline$f_{4}(x)=-\sum_{i=1}^{n} x_{i} \sin \left(\sqrt{\left|x_{i}\right|}\right)$ & $30 / 50$ & {$[-500,500]$} & $\begin{array}{c}- \\
418.9829 * \\
n\end{array}$ \\
\hline $\begin{array}{c}f_{5}(x)=\frac{\pi}{n}\left\{10 \sin ^{2}\left(\pi y_{1}\right)+\sum_{i=1}^{n-1}\left(y_{i}-1\right)^{2}\left[1+10 \sin ^{2}\left(y_{i+1} \pi\right)\right]\right. \\
\left.+\left(y_{n}-1\right)^{2}\right\}+\sum_{i=1}^{n} u\left(x_{i}, 10,100,4\right) *\end{array}$ & $30 / 50$ & {$[-50,50]$} & 0 \\
\hline $\begin{aligned} f_{6}(x)= & (0.1)\left\{\sin ^{2}\left(3 \pi x_{1}\right)+\sum_{i=1}^{n-1}\left(\left(x_{i}-1\right)^{2}\left(1+\sin ^{2}\left(3 \pi x_{i+1}\right)\right)\right)\right. \\
& \left.+\left(x_{n}-1\right)\left(1+\sin ^{2}\left(2 \pi x_{n}\right)\right)\right\}+\sum_{i=0}^{n-1} u\left(x_{i}, 5,100,4\right) *\end{aligned}$ & $30 / 50$ & {$[-50,50]$} & -1.1428 \\
\hline$f_{7}(x)=\sum_{i=0}^{n-1}\left|x_{i}\right|+\prod_{i=0}^{n-1}\left|x_{i}\right|$ & $30 / 50$ & {$[-10,10]$} & 0 \\
\hline$f_{8}(x)=\max \left|x_{i}\right|, \quad 0 \leq i<n$ & $30 / 50$ & {$[-100,100]$} & 0 \\
\hline$f_{9}(x)=\sum_{i=0}^{n-1}\left\lfloor x_{i}+1 / 2\right\rfloor^{2}$ & $30 / 50$ & {$[-100,100]$} & 0 \\
\hline$f_{10}(x)=\left(\sum_{i=0}^{n-1}(i+1) x_{i}^{4}\right)+\operatorname{rand}[0,1]$ & $30 / 50$ & {$[-1.28,1.28]$} & 0 \\
\hline$f_{11}(x)=\sum_{i=0}^{n-1}\left(\sum_{j=0}^{i} x_{i}\right)^{2}$ & $30 / 50$ & {$[-100,100]$} & 0 \\
\hline$f_{12}(x)=\sum_{i=0}^{n-1} 100\left(x_{i+1}-x_{i}^{2}\right)^{2}+\left(x_{i}-1\right)^{2}$ & $30 / 50$ & {$[-30,30]$} & 0 \\
\hline $\begin{aligned} f_{13}(x)=\sin ^{2}\left(3 \pi x_{1}\right) & +\sum_{i=1}^{n-1}\left(\left(x_{i}-1\right)^{2}\left(1+\sin ^{2}\left(3 \pi x_{i+1}\right)\right)\right) \\
& +\left(x_{n}-1\right)\left(1+\sin ^{2}\left(2 \pi x_{n}\right)\right)\end{aligned}$ & $30 / 50$ & {$[-10,10]$} & -21.5024 \\
\hline$f_{14}(x)=\left(\sum_{i=1}^{n} x_{i}^{2}\right)^{1 / 4}\left[\sin ^{2}\left(50\left(\sum_{i=1}^{n} x_{i}^{2}\right)^{1 / 10}\right)+1.0\right]$ & $30 / 50$ & {$[-32.767,32.767]$} & 0 \\
\hline$f_{15}(x)=\frac{1}{n} \sum_{i=1}^{n}\left(x_{i}^{4}-16 x_{i}^{2}+5 x_{i}\right)$ & $30 / 50$ & {$[-5,5]$} & -78.3323 \\
\hline$f_{16}(x)=-\sum_{i=1}^{n} \sum_{j=1}^{5} j \sin \left((j+1) x_{i}+j\right)$ & $30 / 50$ & {$[-10,10]$} & - \\
\hline
\end{tabular}

Remarks 1. Functions sine and cosine take arguments in radians.

Remarks 2. The function $u$ used in $f_{5}$ and $f_{6}$ and the values $y_{i}$ used in $f_{5}$ are all defined below.

$*_{5}: \quad y_{i}=1+\frac{1}{4}\left(x_{i}+1\right)$

$* f_{5}, f_{6}: \quad u(x, a, b, c)=b(x-a)^{c}$ if $x>a, u(x, a, b, c)=b(-x-a)^{c}$ if $x<-a$, else $u(x, a, b, c)=0$ 
Table 2. Numerical results (dimension: 30)

\begin{tabular}{|c|c|c|c|c|c|c|}
\hline \multirow{2}{*}{$\begin{array}{l}\text { Fun- } \\
\text { ction }\end{array}$} & \multicolumn{3}{|c|}{ BPSO } & \multicolumn{3}{|c|}{ QI-PSO } \\
\hline & $\begin{array}{c}\text { Mean Best } \\
\text { Fitness }\end{array}$ & Diversity & Stddev & $\begin{array}{c}\text { Mean Best } \\
\text { Fitness }\end{array}$ & Diversity & Stddev \\
\hline$f 1$ & 81.58668 & $1.075614 \mathrm{e}-08$ & 35.570923 & 0.994954 & $5.948432 \mathrm{e}-11$ & 2.354492 \\
\hline$f 2$ & 2.62144 & $1.696744 \mathrm{e}-96$ & 7.86432 & $2.523604 \mathrm{e}-45$ & $3.203878 \mathrm{e}-20$ & $6.505185 \mathrm{e}-45$ \\
\hline$f 3$ & 0.035265 & $8.958573 \mathrm{e}-08$ & 0.029109 & 0.015979 & $5.454222 \mathrm{e}-08$ & 0.013563 \\
\hline$f 4$ & -8406.742218 & $4.550712 \mathrm{e}-06$ & 595.779702 & -9185.074692 & $4.066819 \mathrm{e}-06$ & 760.633113 \\
\hline$f 5$ & $5.505851 \mathrm{e}-13$ & $1.471375 \mathrm{e}-13$ & $2.757006 \mathrm{e}-25$ & $5.505851 \mathrm{e}-13$ & $7.84124 \mathrm{e}-13$ & $1.913863 \mathrm{e}-25$ \\
\hline f6 & -1.147328 & $4.805696 \mathrm{e}-08$ & 0.003296 & -1.149339 & $3.503531 \mathrm{e}-08$ & 0.003296 \\
\hline f7 & 4.000000 & $7.361003 \mathrm{e}-124$ & 4.89897 & $5.020939 \mathrm{e}-30$ & $9.011109 \mathrm{e}-30$ & $1.271869 \mathrm{e}-29$ \\
\hline$f 8$ & 0.000244 & 0.003937 & 0.000187 & 0.000148 & $7.496987 \mathrm{e}-05$ & $8.128944 \mathrm{e}-05$ \\
\hline$f 9$ & 0.000000 & 1.678432 & 0.000000 & 0.000000 & 0.6674 & 0.000000 \\
\hline$f 10$ & 24.532977 & 0.200206 & 14.640568 & 0.454374 & 0.192434 & 0.353778 \\
\hline$f 11$ & $8.103896 \mathrm{e}-06$ & $1.000654 \mathrm{e}-05$ & $3.623224 \mathrm{e}-06$ & $2.614209 \mathrm{e}-40$ & $1.357432 \mathrm{e}-20$ & $5.848717 \mathrm{e}-40$ \\
\hline$f 12$ & 99.795759 & 3.655268 & 438.491315 & 77.916591 & 0.06907 & 166.009829 \\
\hline$f 13$ & -15.301387 & $5.412045 \mathrm{e}-08$ & 9.36361 & -21.502311 & $6.948980 \mathrm{e}-08$ & $3.552714 \mathrm{e}-15$ \\
\hline$f 14$ & 3.531709 & 0.730954 & 2.484447 & 0.974427 & 0.000265 & 0.283325 \\
\hline$f 15$ & -77.012904 & $1.799400 \mathrm{e}-07$ & 1.049466 & -77.201394 & $1.400101 \mathrm{e}-07$ & 0.565469 \\
\hline$f 16$ & -155.613795 & $1.508819 \mathrm{e}-08$ & 17.664877 & -179.040627 & $1.179486 \mathrm{e}-08$ & 30.28809 \\
\hline
\end{tabular}

Table 3. Numerical results (dimension: 50)

\begin{tabular}{|c|c|c|c|c|}
\hline \multirow{2}{*}{ Function } & \multicolumn{2}{|c|}{ BPSO } & \multicolumn{2}{c|}{ QI-PSO } \\
\cline { 2 - 5 } & Best Fitness & Diversity & Best Fitness & Diversity \\
\hline$f 1$ & 192.383628 & 2.412312 & $4.77049 \mathrm{e}-17$ & $2.596399 \mathrm{e}-09$ \\
\hline$f 2$ & $5.768702 \mathrm{e}-08$ & $1.492921 \mathrm{e}-07$ & $2.161201 \mathrm{e}-73$ & $1.157111 \mathrm{e}-36$ \\
\hline$f 3$ & 0.019661 & $8.376331 \mathrm{e}-08$ & 0.009850 & $8.484164 \mathrm{e}-08$ \\
\hline$f 4$ & -13790.157053 & $5.204209 \mathrm{e}-06$ & -14268.46157 & $6.065605 \mathrm{e}-06$ \\
\hline$f 5$ & 2.535693 & $4.611654 \mathrm{e}-06$ & $3.303511 \mathrm{e}-13$ & $1.506118 \mathrm{e}-13$ \\
\hline$f 6$ & -1.150438 & $6.142788 \mathrm{e}-08$ & -1.150438 & $7.384989 \mathrm{e}-08$ \\
\hline$f 7$ & 9.782400 & $3.776982 \mathrm{e}-15$ & $6.552311 \mathrm{e}-51$ & $2.937678 \mathrm{e}-50$ \\
\hline$f 8$ & 1.514302 & 27.53219 & 0.004083 & 0.017026 \\
\hline$f 9$ & 0.000000 & 1.477857 & 0.000000 & 0.659437 \\
\hline$f 10$ & 110.563669 & 0.245941 & 0.672779 & 0.266817 \\
\hline$f_{11}$ & $7.462966 \mathrm{e}-01$ & $4.993772 \mathrm{e}-05$ & $9.431749 \mathrm{e}-71$ & $2.867124 \mathrm{e}-36$ \\
\hline$f_{12}$ & 22.191719 & 2.324115 & 10.954427 & 0.036353 \\
\hline$f_{13}$ & -11.000000 & $5.633709 \mathrm{e}-08$ & -21.502311 & $8.306594 \mathrm{e}-08$ \\
\hline$f_{14}$ & 10.012826 & 24.758948 & 3.091645 & 0.002222 \\
\hline$f_{15}$ & -77.234331 & $2.398338 \mathrm{e}-07$ & -77.766863 & $3.100701 \mathrm{e}-07$ \\
\hline$f_{16}$ & -204.194616 & $1.54925 \mathrm{e}-08$ & -239.204493 & $1.853805 \mathrm{e}-08$ \\
\hline
\end{tabular}

\section{Experimental Settings}

In order to make a fair comparison of BPSO and QIPSO, we fixed the same seed for random number generation so that the initial swarm population is same for both the algorithms. The number of particles in the swarm (swarm size) is taken as 30. A linearly decreasing inertia weight is used which starts at 0.9 and ends at 0.4 , with the user defined parameters $c_{1}=2.0$ and $\mathrm{c}_{2}=2.0$.

We have also evaluated diversity, an important aspect for checking the efficiency of the swarm, for 
both the algorithms. The diversity measure [14] of the swarm is taken as:

$$
\operatorname{Diversity}(S(t))=\frac{1}{n_{s}} \sum_{i=1}^{n_{s}} \sqrt{\sum_{j=1}^{n_{x}}\left(x_{i j}(t)-\overline{x_{j}(t)}\right)^{2}}
$$

where $S$ is the swarm, $n_{s}=|S|$ is the swarm size, $n_{x}$ is the dimensionality of the problem, $x_{i j}$ is the $j^{\text {th }}$ value of the $i$ 'th particle and $\overline{x_{j}(t)}$ is the average of the $\mathrm{j}^{\text {th }}$ dimension over all particles, i.e.

$$
\overline{x_{j}(t)}=\frac{\sum_{i=1}^{n} x_{i j}(t)}{n_{s}} .
$$

For each algorithm, the maximum number of iterations allowed was set to 30,000 for 30 dimensions and 100,000 for 50 dimensions. For 30 dimensions, a total of 10 runs for each experimental setting were conducted and the average fitness of the best solutions throughout the run was recorded. For 50 dimensions only a single run was performed and the best fitness was recorded. Each algorithm was tested with all of the numerical benchmark problems reported in Table 1.

\section{Experimental Results}

In order to check the compatibility of the proposed QI-PSO algorithm we have tested it on a suite of 16 benchmark problems given in Table 1. The test bed comprises of a variety of problems ranging from a simple spherical function to highly multimodal functions and also a noisy function (with changing optima). In Table 2, we have shown the results of problems with dimension 30 and in Table 3, are given the results of all problems with increased dimension 50 in terms of best fitness value and diversity. In Table 4 and 5, we have shown the performance improvement of QI-PSO with BPSO. Figures 1 - 4 show the mean best fitness curves for the first four benchmark problems.

From the numerical results presented in Tables 2, 3, 4 and 5, it is evident that QI-PSO is a clear winner. It gave a better performance in comparison to the BPSO algorithm for problems with dimension 30 in all the cases except for function $f_{5}$ and $f_{9}$. In this case both BPSO and QI-PSO performs the same. However it is quite interesting to note that QI-PSO gave a much better performance for the same function $\left(f_{5}\right)$ when the dimension is increased to 50. In terms of percentage of improvement (of average fitness function value), the results favor QI-PSO particularly for higher dimension (50).

\section{Conclusions}

In this we proposed a simple and modified version of BPSO by incorporating a newly defined crossover operator in it. The empirical results show that the proposed algorithm is quite competent for solving problems of dimensions up to 50 .

Metaphorically, this article uses the concept of polygamy among various Indian tribes, where the leader of the tribe keeps two partners to produce an offspring.

However, we would also like to say that since the results quoted here are based on the empirical study only and we are yet to explore theoretical relevance of the proposed QI-PSO, making any concrete judgment does not sound fair. Moreover the platform on which we have conducted the experiments is quite narrow. We are working on the problems with higher dimensions (100 and above) and in future we would try more complex optimization problems particularly problems in dynamic environment.

Table 4. Improvement (\%) of QI-PSO in comparison with BPSO (dimension: 30)

\begin{tabular}{|c|c|c|c|}
\hline Function & $\begin{array}{c}\text { Improvement } \\
(\%)\end{array}$ & Function & $\begin{array}{c}\text { Improvement } \\
(\%)\end{array}$ \\
\hline$f 1$ & 98.780495 & $f 9$ & 0.000000 \\
\hline$f 2$ & 100 & $f 10$ & 98.147905 \\
\hline$f 3$ & 54.688785 & $f_{11}$ & 100 \\
\hline$f 4$ & 9.258432 & $f_{12}$ & 21.923946 \\
\hline$f 5$ & 0.000000 & $f_{13}$ & 40.525241 \\
\hline$f 6$ & 0.175277 & $f_{14}$ & 72.409193 \\
\hline$f 7$ & 100 & $f_{15}$ & 0.244751 \\
\hline$f 8$ & 39.344262 & $f_{16}$ & 15.054469 \\
\hline
\end{tabular}

Table 5. Improvement (\%) of QI-PSO in comparison with BPSO (dimension: 50)

\begin{tabular}{|c|c|c|c|}
\hline Function & $\begin{array}{c}\text { Improvement } \\
(\%)\end{array}$ & Function & $\begin{array}{c}\text { Improvement } \\
(\%)\end{array}$ \\
\hline$f 1$ & 100 & $f 9$ & 0.000000 \\
\hline$f 2$ & 100 & $f 10$ & 99.391501 \\
\hline$f 3$ & 49.900819 & $f_{11}$ & 100 \\
\hline$f 4$ & 3.468449 & $f_{12}$ & 50.637321 \\
\hline$f 5$ & 100 & $f_{13}$ & 95.475555 \\
\hline$f 6$ & 0.000000 & $f_{14}$ & 69.123153 \\
\hline$f 7$ & 100 & $f_{15}$ & 0.689502 \\
\hline$f 8$ & 110.877709 & $f_{16}$ & 17.145348 \\
\hline
\end{tabular}




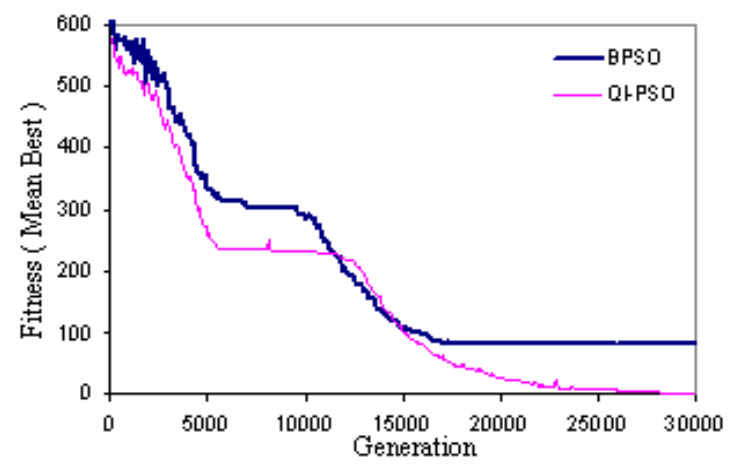

Figure 1. Performance comparison for $f_{l}$

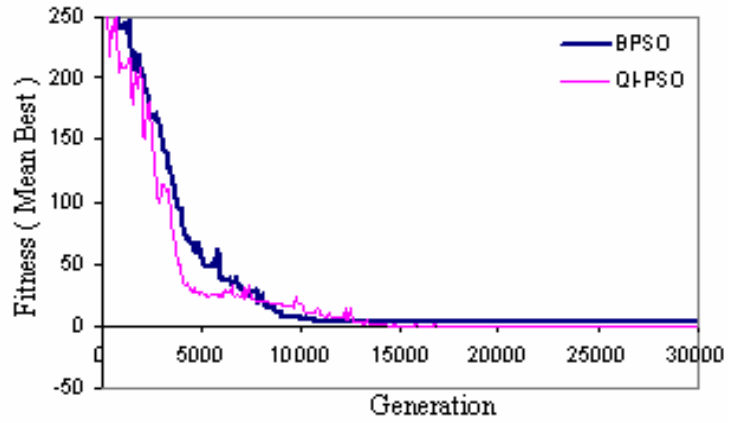

Figure 2. Performance comparison for $f_{2}$

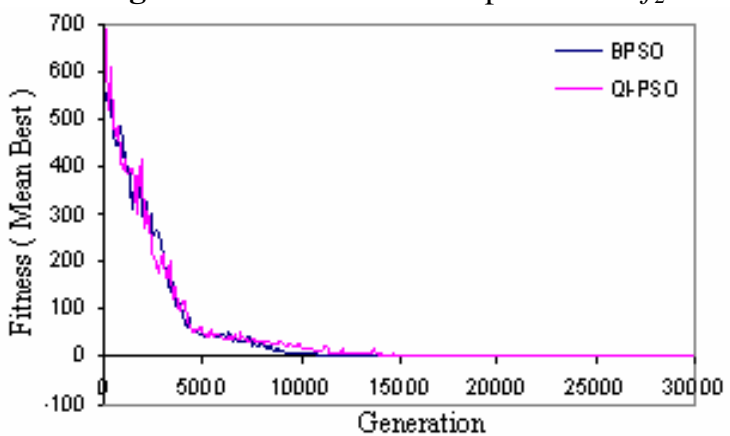

Figure 3. Performance comparison for $f_{3}$

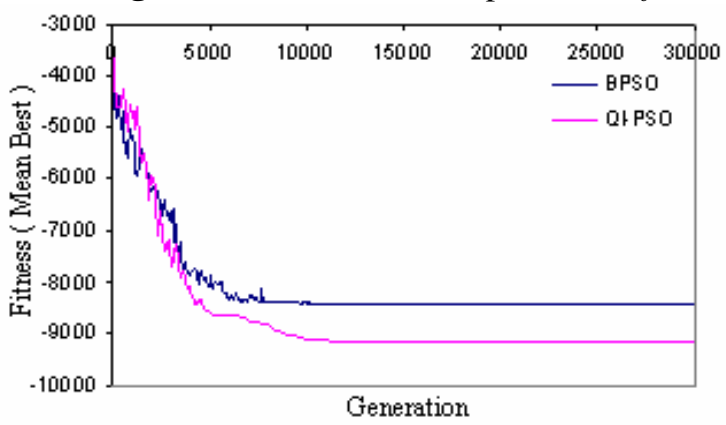

Figure 4. Performance comparison for $f_{4}$

\section{References}

[1] Angeline P. J., "Evolutionary Optimization versus Particle Swarm Optimization: Philosophy and Performance Difference," The 7th Annual Conference on Evolutionary Programming, San Diego,USA,(1998).
[2] X. Hu, R. C. Eberhart, and Y. Shi, "Swarm Intelligence for Permutation Optimization: A Case Study on nQueens problem", In Proc. of IEEE Swarm Intelligence Symposium (2003), pp. 243 - 246.

[3] V. Miranda and N. Fonseca, "EPSO - Best-of-twoworlds Meta-heuristic Applied to Power System problems", In Proc. of the IEEE Congress on Evolutionary Computation, Vol. 2, (2002), pp. 1080 1085.

[4] V. Miranda and N. Fonseca, "EPSO - Evolutionary Particle Swarm Optimization, a New Algorithm with Applications in Power Systems", In Proc. of the Asia Pacific IEEE/PES Transmission and Distribution Conference and Exhibition, Vol. 2, (2002), pp. 745 750.

[5] T-O. Ting, M. V. C. Rao, C. K. Loo, and S-S. Ngu, “A New Class of Operators to Accelerate Particle Swarm Optimization ", In Proc. of the IEEE Congresson Evolutionary Computation, Vol. 4, (2003), pp. 2406 2410.

[6] X. Yao and Y. Liu, "Fast Evolutionary Programming", In L. J. Fogel, P. J. Angeline, and T. B. Back, editors, Proceedings of the Fifth Annual Conference on Evolutionary Programming, MIT Press, (1996), pp. 451 $-460$.

[7] X. Yao, Y. Liu and G.Lin, "Evolutionary Programming made Faster", IEEE Transactions on Evolutionary Computation, Vol. 3(2), (1999), pp. 82 - 102.

[8] P. J. Angeline, "Using Selection to Improve Particle Swarm Optimization", In Proc. of the IEEE Congress on Evolutionary Computation, IEEE Press, (1998), pp. 84 -89 .

[9] M. Clerc, "Think Locally, Act Locally: The Way of Life of Cheap-PSO, an Adaptive PSO”, Technical Report, http: // clerc.maurice.free.fr/pso/, (2001).

[10] Kennedy, J., "The Particle Swarm: Social Adaptation of Knowledge," IEEE International Conference on Evolutionary Computation (Indianapolis, Indiana), IEEE Service Center, Piscataway, NJ,(1997), pg.303308.

[11] R.C. Eberhart, Y. Shi, "Particle Swarm Optimization: developments, Applications and Resources," IEEE International Conference on Evolutionary Computation, (2001), pg. $81-86$.

[12] Shi, Y. H., Eberhart, R. C., "A Modified Particle Swarm Optimizer," IEEE International Conference on Evolutionary Computation, Anchorage, Alaska, (1998), pg. $69-73$.

[13] M. M. Ali and A. Torn, "Population Set Based Global Optimization Algorithms: Some Modifications and Numerical Studies", www.ima.umn.edu/preprints/, (2003).

[14] Engelbrecht, A. P., "Fundamentals of Computational Swarm Intelligence", John Wiley \& Sons Ltd., (2005). 